\title{
Science INSIGHTSB
}

\section{Adhere to the Principle "Primum Non Nocere": A Documentary of the Pioneer Scientist in Labor Pain Control in China \\ Xian Wang}

\author{
Science Insights 2014; 6(2):112-114 \\ Science Insights is published by The Bonoi Academy of Science \& Education, Winston-Salem, NC \\ 27157, USA \\ Copyright (C) 2014 The Bonoi Academy of Science \& Education. All rights reserved.
}

ISSN: 2329-5856

The online version of this article, along with updated information and services, is located on the World Wide Web at:

$\underline{\text { wWw.bonoi.org }}$

Permissions: Requests for permissions to reproduce figures, tables, or portions of articles originally published in Science Insights can be obtained via our Permission Application System, a service of the Copyright Clearance Center. If you cannot access to this system, you can request permission through toour Editorial Office. Once the online version of the published article for which permission is being requested is located, Request Permissions in the middle column of the Web page under Services. Further information about this process is available in the Permissions and Rights Question and Answer document.

Submission: Information about submission to Science Insights is online at: http://www.bonoi.org/node/74 


\section{Adhere to the Principle "Primum Non Nocere": A Documentary of the Pioneer Scientist in Labor Pain Control in China}

By Xian Wang

It is hard to avoid risks during daily medical practice. Even though empiricism hurdles the uniformed and standardized procedures, we are still guided by highgraded clinical trials and professional guidelines. Labor pain is considered as the leading cause for seeking Cesarean section in China, whereas how to reach the ideal labor pain control is still not that popular in the most populous country where most of the parous women are blind to the labor analgesia. Dr. Xiaofeng Shen from Nanjing Medical University leads her team march for the goal of "painless labor delivery" by adhering to the principle "primum non nocere". In collaboration with other domestic and foreign peers, Dr. Shen strives to spread and popularize the knowledge of labor pain control in China, which now has been well-known all over the country for their patient-centered efforts, and has been recognized by Chinese professional and health organizations. For the team of Dr. Shen, the practical principle has been perfected to "primum non nocere, secundus, opinion vulnero"..

How to Cite This Paper: Wang X. Adhere to the principle "primum non nocere": a documentary of the pioneer scientist in labor pain control in china. Science Insights 2014;6(2):112-114.

Keywords: Scientist - Labor analgesia - Xiaofeng Shen - China - Safety

Copyright (C) 2014 The BASE. This is an open access article distributed under the Creative Commons Attribution License, which permits unrestricted use, distribution, and reproduction in any medium, provided the original work is properly cited.

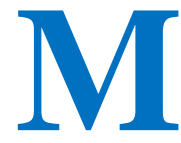

EDICAL practice involves unavoidable risks of which some are definite but some are random. These risks are associated with the unpredictable biology of the individual like genetic and nongenetic variabilities, age, gender, compliance, and environment. How to reach the goal of "primum non nocere", and then "secundus, opinio vulnero" is an art, but also the key principle. Clinical trials and guidelines shape and stand- ardize the approaches and techniques used for caring, but empirical performance makes them be modified to receive the desired favorable outcomes. When new drugs enter our pharmacopoeia or new techniques enrolled into our routine work, some of them were approved by trials but some not. Therefore, our obligation or commission is of vigilant for the possible side effects or adverse events that need to be monitored and reported.

One of the major reasons for the high Cesarean section rate in China is the fear of labor pain. However, labor analgesia is the very technique that can relieve the pain effectively, a technique widely applied in the developed countries. In comparison, various reasons such as difference in understanding, insufficient teaching 


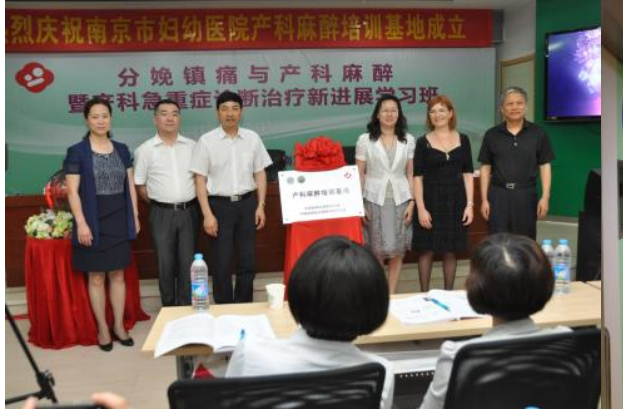

ment, Dr. Shen leads her team march for the goal of "primum non nocere". For them, patient's safety is the first stone no matter what medical technique will be applied in medical practice. For their practical principle, it has been perfected to be "primum non nocere, secundus, opinion vulnero"..

\section{Conflict of Interests}

None

References
1. Wang $F$, Shen X, Guo X, Peng Y, Gu X; Labor Analgesia Examining Group. Epidural analgesia in the latent phase of labor and the risk of cesarean delivery: a five-year randomized controlled trial. Anesthesiology 2009;111(4):871-880..

Dr. Xian Wang, M.D., M.Sc.

Department of Anesthesiology and Critical Care Medicine Nanjing Maternity and Child Health Care Hospital

Nanjing Medical University

Nanjing 210004, China

Tel: $+(0) 86-25-5222-6112$

Fax: $+(0) 86-25-8423-0723$

Email:wangxian2002@126.com 